\title{
Psychological Intervention and COVID-19: What We Know So Far and What We Can Do
}

\author{
Felix Inchausti ${ }^{1,5} \cdot$ Angus MacBeth $^{2} \cdot$ Ilanit Hasson-Ohayon ${ }^{3} \cdot$ Giancarlo Dimaggio $^{4}$
}

Published online: 27 May 2020

(c) Springer Science+Business Media, LLC, part of Springer Nature 2020

\begin{abstract}
The coronavirus COVID-19 and the global pandemic has already had a substantial disruptive impact on society, posing major challenges to the provision of mental health services in a time of crisis, and carrying the spectre of an increased burden to mental health, both in terms of existing psychiatric disorder, and emerging psychological distress from the pandemic. In this paper we provide a framework for understanding the key challenges for psychologically informed mental health care during and beyond the pandemic. We identify three groups that can benefit from psychological approaches to mental health, and/or interventions relating to COVID-19. These are (i) healthcare workers engaged in frontline response to the pandemic and their patients; (ii) individuals who will experience the emergence of new mental health distress as a function of being diagnosed with COVID-19, or losing family and loved ones to the illness, or the psychological effects of prolonged social distancing; and (iii) individuals with existing mental health conditions who are either diagnosed with COVID-19 or whose experience of social distancing exacerbates existing vulnerabilities. Drawing on existing literature and our own experience of adapting treatments to the crisis we suggest a number of salient points to consider in identifying risks and offering support to all three groups. We also offer a number of practical and technical considerations for working psychotherapeutically with existing patients where COVID-19 restrictions have forced a move to online or technologically mediated delivery of psychological interventions.
\end{abstract}

Keywords COVID-19 $\cdot$ Coronavirus $\cdot$ Psychotherapy $\cdot$ Digital therapy $\cdot$ Psychological interventions

\section{Psychological Implications of Coronavirus}

The Coronavirus 2019 (COVID-19) is a newly emergent infectious disease caused by the novel severe acute respiratory syndrome coronavirus 2 (SARS-CoV-2) virus, originated in December 2019 from mainland China, with initial

Felix Inchausti

finchausti@riojasalud.es

1 Department of Mental Health, Servicio Riojano de Salud, Logroño, Spain

2 Centre for Applied Developmental Psychology, Clinical and Health Psychology, School of Health in Social Science, University of Edinburgh, Edinburgh, Scotland

3 Department of Psychology, Bar-Ilan University, Ramat-Gan, Israel

4 Centro Di Terapia Metacognitiva Interpersonale, Rome, Italy

5 Centro de Salud Espartero, Unidad de Salud Mental Infanto-Juvenil, Avda. Pío XII, 12 Bis, 26003 Logroño, La Rioja, Spain cases emerging from the city of Wuhan, Hubei Province (CDCP 2020; Li et al. 2020). Although most individuals diagnosed with COVID-19 present with mild to moderate respiratory symptoms, a substantially minority present with severe symptomatology, with accompanying need for hospital treatment, a further proportion needing intensive care unit (ICU) admission, and an elevated fatality rate. Risk of mortality follows a clear age gradient (Verity et al. 2020). On 30th January 2020, World Health Organization (WHO) officially declared the COVID-19 epidemic as a public health emergency of international concern, followed by designation as a pandemic on 11th March (i.e., presence of illness across multiple continents). The rapid spread of COVID-19 places huge strain on capacity, responsiveness and resilience of public and private healthcare systems worldwide (Emanuel et al. 2020; Legido-Quigley et al. 2020). Across multiple countries this has been accompanied by implementation of public health policies significantly altering everyday life, such as the quarantine of citizens for significant periods of 
time, with both short- and longer-term consequences for psychological distress and wellbeing (Brooks et al. 2020).

At time of writing, the worldwide cases of COVID-19 are steadily increasing across all continents. On 11th April 2020 , the cumulative total of individuals presenting with confirmed COVID-19 was $1,648,365$ people, with a total of 102,216 deaths (WHO 2020). In many countries testing is limited to hospitalised cases, therefore these numbers are likely to significantly underestimate the true prevalence of COVID-19 in the population, given they do not cover mild presentation and asymptomatic cases. There is emerging evidence of the psychological impact of COVID-19 on populations, both directly due to the distress accompanying confirmed cases in individuals and their loved ones, and indirectly due to population health interventions such as quarantine. However, it should be emphasized that the majority of people are not expected to suffer from mental disorders emerging from the pandemic and its impact (Taylor 2019). However, a significant percentage will experience intense emotional adjustment reactions, including fear of contagion (Zhou 2020), impact of prolonged quarantine (Brooks et al. 2020; Xiao 2020), the death of relatives (Wang et al. 2020), or increased social adversity as a consequence of geopolitical instability to civil society associated with the economic crisis (Silva et al. 2018). In China, a survey of 1210 people found that $53.8 \%$ assessed the psychological impact of the situation as moderate-severe, $16.5 \%$ reported moderate to severe depressive symptoms, $28.8 \%$ moderate to severe anxiety symptoms, and $8.1 \%$ moderate to severe stress levels. Most respondents (84.7\%) spent between 20 and $24 \mathrm{~h}$ a day confined at home and the main concern (75.2\%) was that his/her relatives would become infected with COVID-19 (Wang et al. 2020).

Based on our survey of preliminary current research and on previous literature on coping with past coronavirus-based epidemics (e.g. Severe Acute Respiratory Syndrome, SARS; and Middle East Respiratory Syndrome, MERS) we identify three groups at risk for psychological morbidity during and after the COVID-19 pandemic.

The first group are healthcare professionals, particularly those working in inpatient physical health settings, who experience higher frequency of exposure to the virus and higher viral load in the workplace; compounded by significantly increased workload, high risk procedures and the low availability of necessary personal protective equipment (PPE). Thus, health professionals are at risk of elevated levels of depression, anxiety and sleep disorders ( $\mathrm{Li}$ et al. 2020), and many among them harbour fears of being infected during work shifts. Recent findings on medical students in the current crisis supporting this (Al-Rabiaah et al. 2020). This is also in line with previous experiences from SARS/ MERS, showing frontline health professionals constitute a unique risk group, especially after pandemic containment ends and systems move towards mitigation of the disease impact (Gardner and Moallef 2015; Lee et al. 2018). Of note, many other workers are exposed to the same risk and fear of contagion, such as police officer, postal carrier, emergency medical technicians or trash collectors.

The second elevated risk group that should be considered include individuals who, as a result of the crisis, have been exposed to potentially traumatic events such as loss of a loved one, threats to one's health and to the ability to work and make a living, and concerns about their future capacity to maintain a sufficient income. These people may express symptoms of post-traumatic stress disorder (PTSD), depression or complicated grief disorder, consistent with the literature on psychological and psychiatric sequelae of global emergencies or disasters (Goldmann and Galea 2014). This group may not emerge immediately within the pandemic, and presentations may only become apparent after several months, even after the incidence of COVID-19 has peaked.

A third group of people at increased risk for psychological problems consists of people with pre-existing psychopathology, especially those with severe or complex psychiatric disorders. Their existing presentation may be exacerbated by extreme isolation due to exposure to either the virus or associated social distancing. In this sense, social distancing may exacerbate existing social isolation in this vulnerable group. There is conflicting evidence from previous studies on the responses of people with severe psychiatric disorders to different types of disasters such as earthquakes, with some evidence for higher levels of avoidance-related coping being associated with higher distress (Horan et al. 2007), but other studies showing that this risk is somewhat disorder specific with pre-disaster mood and anxiety disorders, but not psychotic disorders, predicting further psychological distress (Katz et al. 2002). This group also includes individuals with more common psychopathologies (e.g. depression and anxiety) who were receiving primary care mental, health treatment or psychotherapy prior to the onset of COVID-19 restrictions. Other people exposed to psychological suffering are those who have to live alone during the quarantine, who has been recently bereaved by the coronavirus, but the bereavement process has been disrupted by the lockdown, and ones that are not allowed to visit their loved ones who are in hospital for whatsoever medical conditions.

\section{Assisting Frontline Health Professionals and COVID-19 Diagnosed Patients}

As Duan and Zhu (2020) highlight, specialized psychological intervention for COVID-19 should be dynamic and flexible enough to adapt quickly to the different phases of the pandemic. In the early stages, clinical psychologists, psychotherapist and psychological intervention specialists should 
actively collaborate with the rest of the multi-professional healthcare system in the treatment of the immediate impacts of COVID-19 presentations (Mohammed et al. 2015). This may take the shape of organising or enabling healthcare systems to orientate towards psychological impacts of a pandemic, facilitate public mental health approaches to increasing population awareness of mental health; or organizing systems for psychologically informed interventions. This may also include task-shifting of psychological interventions either to delivery through digital means, or by different professional groups. Potential therapeutic targets include:

1. Training and support for health professionals at 'high exposure risk' to identify and manage emotional reactions, that may hinder their clinical work in frontline health delivery. This includes, for instance, managing anxiety, fear of contagion, episodes of acute stress or promoting self-care/reducing burnout. The main objective of this approach is to maximise psychological resilience in as many professionals as possible who have frontline duties during a pandemic (Chen et al. 2020). Importantly, in the peak of a pandemic, interventions such as psychological debriefing, critical incident stress debriefing or any other single session intervention mandating staff to talk about their thoughts or feelings are not recommended. That said, compassionate and sensitive awareness of the impact of critical care on health care professionals can be used to facilitate one on one support, should that person wish it (NICE 2018).

2. Next it is important to engage emotionally vulnerable groups, especially people with previous psychopathology. The main goal here is to support individuals undergoing COVID-19 treatment or preventative quarantine. The mental health symptoms of this group of patients with COVID-19 should also be monitored, although the presence of non-essential professionals such as psychiatrists, clinical psychologists or social mental health workers in isolation rooms for COVID-19 patients is completely discouraged. Therefore, front-line psychological support either needs to be facilitated by medical staff involved in immediate care (which may not be possible if the health system is at capacity) or be implemented indirectly through telecare systems. Serious psychiatric emergencies such as aggression, self-harm or suicide attempts will still need to be addressed in person. For patients with acute symptomatology and diagnosed or suspected COVID-19, professionals who assist them face-to-face should be protected to minimize the risk of contagion (e.g. via appropriate PPE) and ensure both their safety and that of the patient.

All other outpatient psychological interventions can be effectively carried out by digital care. Phone and internet enabled psychological interventions have been demonstrated to be clinically effective in a wide variety of mental disorders (Irvine et al. 2020). Related to this, it is also important to tailor standard mental health delivery for individuals with pre-existing psychiatric disorders to acknowledge the impact of social isolation and distancing on mental health as part of adaptation to 'life under lockdown' or quarantine.

3. Relatives of patients admitted by the coronavirus in a severe condition, poorly prognosed or who have already died. In such interventions it is essential not to pathologize the normal emotional reactions of family members and it is important to establish clear and consensual criteria with all the professionals involved to determine whether intervention is more beneficial than not to do so (von Blanckenburg and Leppin 2018).

As the pandemic plateaus, and societies begin to emerge from distancing, mental health symptoms such as hypochondriasis, anxiety, insomnia or acute stress, as well as symptoms consistent with PTSD are expected to present across health systems. In these cases, the first-line intervention should be psychological, minimizing as far as possible the use of drugs (NICE 2014, 2018). Furthermore, the literature emphasizes the importance of not starting formal psychological treatments quickly and without careful assessment, including active monitoring. As noted above, although well intentioned, intervening in individual's natural coping mechanisms too early can be detrimental. There is evidence that these interventions may be ineffective or even increase the likelihood of developing PTSD (NICE 2018). Special attention should also be paid to: potential for "re-traumatization" of PTSD presentations where trauma-focused therapies are implemented without adequate psychotherapeutic frameworks and structures (Duckworth and Follette 2012); and guarding against the development of interventions for those that have recovered from COVID-19 that stigmatize or block access of the to a new functional identity as survivors of the pandemic (Muldoon et al. 2019). Going forward it is also crucial to ensure individuals affected by COVID-19 retain a sense of their overall identity, and that this is not subsumed into an explanatory model reduced to the illness.

Any intervention should be based on a thorough assessment of possible risk factors that may maintain the problem, the patient's prior state of mental health, the history of bereavement, the presence of a history of self-harm or suicidal behaviours in both the patient and his/her family, the history of previous traumas, and the socio-economic context of the patient. At this stage, it is also important to recognise the likely profound impact of COVID-19 on economic, social, and political levels at all levels from the individual to international. This may, therefore, require mental health systems to adopt new ways of working with structural inequalities emerging from the aftermath of COVID-19 and 
consistent with a social determinants of mental health model (e.g., Lund et al. 2018).

\section{Current and Emerging Challenges from the Pandemic}

In organizing psychological assistance within and across various stages of the pandemic, we highlight four major challenges:

1. Healthcare system deficits, both in terms of material and human resources (i.e., lack of adequate PPE, infrastructure for digital interventions, staffing) or in mental health professionals not specialized in the psychological approach of crises and emergencies (Shultz et al. 2015; Shultz and Neria 2013). In China, the scarcity of human resources led to individual professionals accumulating multiple responsibilities, reducing the effectiveness of their interventions (Duan and Zhu 2020). For this reason, government, policy makers and health managers need to be aware of health systems strengthening for increasing the capacity of mental health professionals, facilitate training for emergency intervention, and monitor workload burdens, especially when sustained over time.

2. Societal underestimation of the (short- and long-term) psychological consequences of pandemics and, consequently, limited resources to cope with them (Bitanihirwe 2016). There is evidence that individuals exposed to public health emergencies have increased psychopathological vulnerability both during and after the potentially traumatic event (Fan et al. 2015). Although the international COVID-19 pandemic response has been unprecedented in terms of mobilisation of resource and finance, there will also be long-term impacts in terms of treatment burden, including mental health, particularly in low resource and conflict settings (UN 2020). In China, the progression of COVID-19 aggravated the mental health of infected patients, the general population and health professionals (Duan and Zhu 2020). Therefore, it is important to evaluate and identify all risk groups and adapt interventions to their specific needs. Among the variables to consider are disease trajectory, severity of clinical symptoms, place of treatment (inhome or out-of-home isolation, ICU, etc.), history of previous trauma and, previous history of mental health problems. Having this information will help classify people at risk and enable specific preventive mental health measures to be put in place.

3. Poor planning and coordination of psychological interventions, especially when they are applied at different levels and by different professionals (Zhang et al. 2020).
In China, at the start of the COVID-19 outbreak, the absence of adequate planning of psychological interventions led to fragmented or disorganized implementation, compromising effectiveness and efficacy, and hampering access to available health resources. Any psychological intervention should be planned and coordinated together with all the social-health stakeholders involved, particularly primary healthcare services and specialized mental health services. This maximised the potential for adequate continuity of care even after acute phase of the pandemic recedes.

4. Finally, there is also a risk attached to early crisis responses, leading to a proliferation of interventions and frameworks associated with an oversupply of well-intentioned but potentially non-evidence based, psychological assistance, often non-governmental organizations (NGO) and the third sector. This is not to say all NGO interventions are compromised, and indeed prevention in mental health is highly desirable. That said, delivery of preventive interventions must be balanced by delivery and/or supervision applied by appropriately qualified professionals (Loewenstein 2018; Ogden 2019).

\section{Existing Caseload: "How to" Deliver Technology Enabled Therapy Under Lockdown}

As previously noted, where health systems have sufficient flexibility, for those with existing mental health conditions should continue their psychological interventions by technology enabled means. This can include telephone consults, or increasingly via digital platforms such as Skype, Zoom or health provider developed platforms. This presents a number of specific challenges including familiarity with the technology (both therapist and client), adaptation of the therapeutic intervention, awareness of the additional parameters of delivering therapy in lockdown conditions, and the accompanying question of the purpose of therapy in such unusual circumstances.

There are thus several difficulties that psychotherapists and practitioners have in adjusting their practice to technology enhanced therapy, which is now delivered from their own homes, as opposed to familiar public facilities or private practices. The following suggestions of how to adapt psychotherapy to this unique condition have emerged from our everyday clinical experiences over the adaptation to lockdown in several countries, and represent an attempt to systemize clinical practice for the duration of the emergence and of social life restrictions. Therefore, we provide a number of key points to guide clinicians in adapting practice. 
- Draft a new contract. Many patients will have difficulties in accepting digital psychotherapy. Clinicians must be clear that this is pragmatically the only option available (if this is the case), but also acknowledge and selfregulate their own difficulties with changes such as worry for the client's mental health, irritation with the option of discontinuing face to face psychotherapy or guilt at the idea of not being available enough. In all of these cases the clinician remains open for phone/video contact where the patient experiences psychological problem, but negotiation is required over whether sessions are for crisis-management only; or whether regular sessions are still possible and/or desirable to both parties. This can help retain a balance between acceptance of difficulties and the maintenance of a robust treatment framework.

- Raise the bar for what we consider psychopathology. Reactions of distress, such as fear, rage, anxiety, obsessions, guilt, constriction, rebellion against authority, emotion and behavioural dysregulation, albeit transitory, are to a certain extent normal during a crisis. The clinician must first and foremost help the patients understand that their suffering is human and mostly unavoidable, this is not to say that they should be ignored or minimised. When patients can note how their mind is overwhelmed by symptoms, affect or relational problems, this creates a basis for agreement to work on them.

- Common factors (e.g., Norcross and Lambert 2019) are even more important than usual. In particular, we think that validation, sharing and self-disclosure become of uttermost importance. Validation follows from the above, that adjustment to the "new normal' is normal and patients experience is human. Therapists can note how experiencing fears for their own and their loved ones health is understandable, that to be worried about the future of the economy is reasonable, how to behave with a certain degree of obsessions is adaptive (e.g. hand hygiene) or that unexpected losses of temper are to be expected in confinement. Where sharing is appropriate, the clinician may provide examples of witnessing the same experiences and noting this is part of what the humanity is experiencing now. This is aimed at reducing feelings of self-shaming, self-criticism stigma, or guilt for one's own weaknesses. Self-disclosure is unique in this aspect. Above all, it is one of the most powerful interventions (Safran and Muran 2000) and in this moment becomes even more necessary. Therapists may need to strategically disclose moments of their own personal vulnerability during the outbreak. We contend that in this moment clinicians should mindfully and tactically not stick to one of the principles of good self-disclosures (e.g., Dimaggio et al. 2015), that is clinicians should disclose well-regulated feelings and thoughts. In this moment, still having command over their own experi- ences, clinicians may disclose moments in which they experienced momentarily feelings of fear, even moving closer to panic, worry, anger, sadness, rebellion and irritation than one ordinarily would. This helps create a sense of human connection and reduces in session risk, on the client's side of self-blaming or setting unrealistic standards of good mental health for the self (Safran and Muran 2000; Inchausti et al. 2019). This can be balanced in session with learning from these experiences of momentary dysregulation.

- Create the therapeutic environment. We are not working in our offices but often from our homes. The therapy space must be therefore be created anew. For video-therapy the clinician should choose what part of their home they want to show beyond their shoulders and possibly consider the patients' personality. Equally, the therapists will be projecting a sense of their own identify in these choices. With some patients it is better to choose a more neutral/professional background, for example bookshelves or a working table. With other patients there is less this need, and they experience a sense of familiarity even when they see the kitchen of the windows of the therapists' home. In any case, asking patients for feedback about how they experience the therapist in this new environment is crucial. Another issue is how to present oneself in the camera. Absence of embodied intersubjectivity deprives the session of face-to-face aspects of the human connection. We consider that adjusting zoom of the webcam, which means placing oneself at some distance can be helpful. Showing only one's face is artificial and deprives the client of gestures and nonverbal markers from the therapist. Conversely, at least a halflength shot (e.g. breaking news conductors) is better and some background must be present, so the patients retains a sense of a human being in context. This way therapists can use arms and hands and chest and shoulders to convey nonverbal signals making communication more natural. Alternatively, some patients may feel more comfortable without using a camera and the use of audio might suit them better. Coping with such anxiety disorders as social anxiety might lead patients to avoid video. As in any form of coping, if using video is too much of an emotional burden to that client, the clinician accepts phone consultation, but keeps exploring the possibility to switch to video, which would be a kind of behavioural exposure. A compromise would be using a web platform with video disabled. Simply accepting coping deprives the clinician the possibility to counteract psychopathology. Whereas, gently asking if the patient feels ready to switch to video, and explore the cognitive-affective antecedents of the possible refusal gives precious information about residual maladaptive interpersonal schemas which are one fundamental therapy target. 
- Help patients build their own environment. Clinicians may offer suggestions for how to create a therapeutic space, safe and protected from interference. Of course, having a private, distraction-free room is best, but even in this case patients can be suggested to use headphones and a microphone, and maybe some background music, so reducing the risk others listen. Alternatively, sessions can be conducted over smartphone in the open, for example a private garden, the parking lot or one's car. Trivial as they may sound, we have found these suggestions help many patients to accept and practice therapy even after initial reluctance.

- Therapeutic focus -only self-regulation and overcoming distress or exploration of opportunities for building healthy parts and pursuing autonomy, exploration and expanding the healthy self (Dimaggio et al. 2015). We have noted that in majority of cases where we have adjusted delivery of psychotherapy to fit the pandemic restrictions, patients are seeking a balance between acceptance of the current condition, whilst still trying to challenge maladaptive schemas and develop an emergent healthy part of the self. Indeed, once issues relating to the present crisis have been dealt with, patient and therapist may explore how the current distressing conditions create suffering not only for their direct traumatic effects, but also because they may indirectly bring existing personality, cognitive and emotional vulnerabilities to the fore. Thus, clinicians may help the patients connect their present experiences to lifelong vulnerabilities, enabling therapeutic work to continue as they did before the emergency, albeit with specific adaptations. For example, prior to lockdown patients with avoidant personality disorders may have started questioning schemas of themselves as inferior and others are judging and therefore, they coped with social avoidance (Inchausti et al. 2018). In this moment behavioural experiments aimed at increasing social contact and thus further challenging the schemas are more difficult to enact. Yet, the clinician may still explore opportunities, and build more basic steps for future real-life exposures. Patients looking for employment may be able to access online courses or training for life after. Patients searching for romantic partner may use dating Apps or explore the feelings and thoughts they experience when chatting with some new acquaintance. Even the home may be a test ground for new experiments. One client related difficulty in showing personal vulnerabilities to significant persons because she had learned that if she revealed these emotions others either became unavailable or distressed; therefore, she had avoided disclosure, or felt guilty for burdening them. Lockdown and having to live with her partner 24:7 helped her realize that there was no point in her concealing her personal feelings, thus she burst into tears with her partner; relating afterwards in therapy that she felt relieved as she realized that that was possible. This enabled schema-driven difficulties in continuing with disclosure of feelings could be addressed as a current therapeutic issue.

Finally, some practices like two-chairs, sensorimotor work, guided imagery exercises, can regularly be performed simply adjusting the zoom in the patient room. The therapists may ask the client to step back so the whole body can be observed and then ask to close their eyes and engage in guided imagery, or use bodily oriented work like grounding (Lowen 1971) to enhance self-regulation or connecting with feelings of strength and personal agency. That said, for some patients that are unwilling or do not want to use this platform for treatment. If they are content to postpone specific elements of treatment until restrictions are lifted, the therapist should be sensitive in recognizing distress but also respecting the decision-making process. It is still possible to remain open to the patient recontacting the therapist to recommence therapy.

\section{Conclusion}

To conclude, the COVID-19 pandemic and associated disruption to society poses major challenges to the provision of mental health services. These challenges include the need to identify and monitor possible risk groups for psychological morbidity as well as exploring new ways of providing services. As a heuristic, it is useful to consider three (potentially overlapping) groups that can benefit from psychological frameworks for mental health, and/or treatment approaches. These are (i) healthcare workers engaged in frontline response to the pandemic and their patients; (ii) individuals who will experience the emergence of new mental health distress as a function of being diagnosed with COVID-19, or losing family and loved ones to the illness, or the psychological effects of prolonged social distancing; and (iii) individuals with existing mental health conditions who are either diagnosed with COVID-19 or whose experience of social distancing exacerbates existing vulnerabilities. There are yet limited data on the mental health impacts of the current crisis, but evidence from past epidemics (e.g., MERS and SARS) offer a basis for identifying risk groups and preparing management strategies. The current crisis is the first global crisis in the age of mass internet supported communication, and this offers opportunities and challenges for delivering high-quality psychological therapies online. Practical and technical adjustments to therapy can and have already been made, but as the pandemic unfolds it will be important to generate a corpus of knowledge both on the effectiveness of technologically supported psychotherapy, 
and to share technique in working with patients in an environment where technological changes intersect with societal changes due to the pandemic.

\section{Compliance with Ethical Standards}

Conflict of interest We have no conflicts of interest to disclose.

\section{References}

Al-Rabiaah, A., Temsah, M.-H., Al-Eyadhy, A. A., Hasan, G. M., AlZamil, F., Al-Subaie, S., et al. (2020). Middle East Respiratory Syndrome-Corona Virus (MERS-CoV) associated stress among medical students at a university teaching hospital in Saudi Arabia. Journal of Infection and Public Health.. https://doi.org/10.1016/j. jiph.2020.01.005.

Bitanihirwe, B. K. Y. (2016). Monitoring and managing mental health in the wake of Ebola. Annali dell'Istituto Superiore Di Sanita, 52(3), 320-322. https://doi.org/10.4415/ANN_16_03_02.

Brooks, S. K., Webster, R. K., Smith, L. E., Woodland, L., Wessely, S., Greenberg, N., et al. (2020). The psychological impact of quarantine and how to reduce it: Rapid review of the evidence. The Lancet, 395(10227), 912-920. https://doi.org/10.1016/S0140 $-6736(20) 30460-8$.

Centers for Disease Control and Prevention (CDCP). (2020). Coronavirus disease 2019 (COVID-19). Retrieved March 26, 2020, from https://www.cdc.gov/coronavirus/about/index.html.

Chen, Q., Liang, M., Li, Y., Guo, J., Fei, D., Wang, L., et al. (2020). Mental health care for medical staff in China during the COVID19 outbreak. Lancet Psychiatry, 7(4), e15-e16. https://doi. org/10.1016/S2215-0366(20)30078-X.

Dimaggio, G., Montano, A., Popolo, R., \& Salvatore, G. (2015). Metacognitive interpersonal therapy for personality disorders: A treatment manual. London: Routledge. https://doi.org/10.4324/97813 15744124.

Duan, L., \& Zhu, G. (2020). Psychological interventions for people affected by the COVID-19 epidemic. Lancet Psychiatry, 7(4), 300-302. https://doi.org/10.1016/S2215-0366(20)30073-0.

Duckworth, M. P., \& Follette, V. M. (Eds.). (2012). Retraumatization: Assessment, treatment, and prevention. London: Routledge/Taylor \& Francis Group.

Emanuel, E. J., Persad, G., Upshur, R., Thome, B., Parker, M., Glickman, A., et al. (2020). Fair allocation of scarce medical resources in the time of COVID-19. New England Journal of Medicine. https://doi.org/10.1056/NEJMsb2005114.

Fan, F., Long, K., Zhou, Y., Zheng, Y., \& Liu, X. (2015). Longitudinal trajectories of post-traumatic stress disorder symptoms among adolescents after the Wenchuan earthquake in China. Psychological Medicine, 45(13), 2885-2896. https://doi.org/10.1017/S0033 291715000884.

Gardner, P. J., \& Moallef, P. (2015). Psychological impact on SARS survivors: Critical review of the English language literature. Canadian Psychology/Psychologie Canadienne, 56(1), 123-135. https://doi.org/10.1037/a0037973.

Goldmann, E., \& Galea, S. (2014). Mental health consequences of disasters. Annual Review of Public Health, 35(1), 169-183. https ://doi.org/10.1146/annurev-publhealth-032013-182435.

Horan, W. P., Ventura, J., Mintz, J., Kopelowicz, A., Wirshing, D., Christian-Herman, J., et al. (2007). Stress and coping responses to a natural disaster in people with schizophrenia. Psychiatry
Research, 151(1-2), 77-86. https://doi.org/10.1016/j.psych res.2006.10.009.

Inchausti, F., Prado-Abril, J., Sánchez-Reales, S., Vilagrà-Ruiz, R., \& Fonseca-Pedrero, E. (2018). El trastorno de personalidad por evitación: una propuesta de tratamiento especializado en la sanidad pública española [The avoidant personality disorder: A proposal for specialized treatment in the Spanish National Health System]. Ansiedad y Estrés, 24(2-3), 144-153. https:// doi.org/10.1016/j.anyes.2018.05.002.

Inchausti, F., Sánchez-Reales, S., \& Prado-Abril, J. (2019). Proceso psicoterapéutico en los trastornos del espectro psicótico [Psychotherapeutic process in psychotic spectrum disorders]. In E. Fonseca-Pedrero (Ed.), Tratamientos psicológicos para la psicosis [Psychological treatments for psychosis] (pp. 145180). Pirámide Editorial.

Irvine, A., Drew, P., Bower, P., Brooks, H., Gellatly, J., Armitage, C. J., et al. (2020). Are there interactional differences between telephone and face-to-face psychological therapy? A systematic review of comparative studies. Journal of Affective Disorders, 265, 120-131. https://doi.org/10.1016/j.jad.2020.01.057.

Katz, C. L., Pellegrino, L., Pandya, A., Ng, A., \& DeLisi, L. E. (2002). Research on psychiatric outcomes and interventions subsequent to disasters: A review of the literature. Psychiatry Research, 110(3), 201-217. https://doi.org/10.1016/s0165 -1781(02)00110-5.

Lee, S. M., Kang, W. S., Cho, A.-R., Kim, T., \& Park, J. K. (2018). Psychological impact of the 2015 MERS outbreak on hospital workers and quarantined hemodialysis patients. Comprehensive Psychiatry, 87, 123-127. https://doi.org/10.1016/j.compp sych.2018.10.003.

Legido-Quigley, H., Mateos-García, J. T., Campos, V. R., Gea-Sánchez, M., Muntaner, C., \& McKee, M. (2020). The resilience of the Spanish health system against the COVID-19 pandemic. Lancet Public Health. https://doi.org/10.1016/S2468-2667(20)30060-8.

Li, Q., Guan, X., Wu, P., Wang, X., Zhou, L., Tong, Y., et al. (2020). Early transmission dynamics in Wuhan, China, of novel coronavirus-infected pneumonia. New England Journal of Medicine. https ://doi.org/10.1056/NEJMoa2001316.

Loewenstein, R. J. (2018). Dissociation debates: Everything you know is wrong. Dialogues in Clinical Neuroscience, 20(3), 229-242.

Lowen, A. (1971). The language of the body. London: Macmillan General Reference.

Lund, C., Brooke-Sumner, C., Baingana, F., Baron, E. C., Breuer, E., Chandra, P., et al. (2018). Social determinants of mental disorders and the Sustainable Development Goals: A systematic review of reviews. Lancet Psychiatry, 5(4), 357-369. https://doi. org/10.1016/S2215-0366(18)30060-9.

Mohammed, A., Sheikh, T. L., Poggensee, G., Nguku, P., Olayinka, A., Ohuabunwo, C., et al. (2015). Mental health in emergency response: Lessons from Ebola. The Lancet, 2(11), 955-957. https ://doi.org/10.1016/S2215-0366(15)00451-4.

Muldoon, O. T., Haslam, S. A., Haslam, C., Cruwys, T., Kearns, M., \& Jetten, J. (2019). The social psychology of responses to trauma: Social identity pathways associated with divergent traumatic responses. European Review of Social Psychology, 30(1), 311-348. https://doi.org/10.1080/10463283.2020.1711628.

National Institute for Health and Care Excellence (NICE). (2014). Anxiety disorders. Quality standard. Retrieved March 26, 2020, from www.nice.org.uk/guidance/qs53.

National Institute for Health and Care Excellence (NICE). (2018). Posttraumatic stress disorder. NICE guideline. Retrieved March 26, 2020, from www.nice.org.uk/guidance/ng116.

Norcross, J. C., \& Lambert, M. J. (Eds.). (2019). Psychotherapy relationships that workevidence-based therapist contributions (3rd ed.). Oxford: Oxford University Press. https://doi.org/10.1093/ med-psych/9780190843953.001.0001. 
Ogden, J. (2019). Do no harm: Balancing the costs and benefits of patient outcomes in health psychology research and practice. Journal of Health Psychology, 24(1), 25-37. https://doi. org/10.1177/1359105316648760.

Safran, J. D., \& Muran, J. C. (2000). Negotiating the therapeutic alliance: A relational treatment guide. New York: Guilford Press.

Shultz, J. M., Baingana, F., \& Neria, Y. (2015). The 2014 Ebola outbreak and mental health: Current status and recommended response. Journal of the American Medical Association, 313(6), 567-568. https://doi.org/10.1001/jama.2014.17934.

Shultz, J. M., \& Neria, Y. (2013). Trauma signature analysis. Disaster Health, 1(1), 4-8. https://doi.org/10.4161/dish.24011.

Silva, M., Resurrección, D. M., Antunes, A., Frasquilho, D., \& Cardoso, G. (2018). Impact of economic crises on mental health care: A systematic review. Epidemiology and Psychiatric Sciences, 29, e7. https://doi.org/10.1017/S2045796018000641.

Taylor, S. (2019). The psychology of pandemics. Preparing of the next global outbreak of infectious disease. Cambridge: Cambridge Scholars Publishing.

United Nations (UN). (2020). COVID-19 Global Humanitarian Response Plan. Retrieved April 2, 2020, from https://www.unesc ap.org/news/covid-19-global-humanitarian-response-plan.

Verity, R., Okell, L. C., Dorigatti, I., Winskill, P., Whittaker, C., Imai, N., et al. (2020). Estimates of the severity of COVID-19 disease. MedRxiv.. https://doi.org/10.1101/2020.03.09.20033357.

von Blanckenburg, P., \& Leppin, N. (2018). Psychological interventions in palliative care. Current Opinion in Psychiatry, 31(5), 389-395. https://doi.org/10.1097/YCO.0000000000000441.

Wang, C., Pan, R., Wan, X., Tan, Y., Xu, L., Ho, C. S., et al. (2020). Immediate psychological responses and associated factors during the initial stage of the 2019 Coronavirus Disease (COVID-19) epidemic among the general population in China. International Journal of Environmental Research and Public Health, 17(5), 1729. https://doi.org/10.3390/ijerph17051729.

World Health Organization (WHO). (2020). Coronavirus disease (COVID-19) technical guidance: Infection prevention and control/ WASH. Retrieved April 2, 2020, from https://www.who.int/emerg encies/diseases/novel-coronavirus-2019/technical-guidance/infec tion-prevention-and-control.

Xiao, C. (2020). A novel approach of consultation on 2019 novel coronavirus (COVID-19)-related psychological and mental problems: Structured letter therapy. Psychiatry Investigation, 17(2), 175176. https://doi.org/10.30773/pi.2020.0047.

Zhang, J., Wu, W., Zhao, X., \& Zhang, W. (2020). Recommended psychological crisis intervention response to the 2019 novel coronavirus pneumonia outbreak in China: A model of West China Hospital. Precision Clinical Medicine. https://doi.org/10.1093/ pcmedi/pbaa006.

Zhou, X. (2020). Psychological crisis interventions in Sichuan Province during the 2019 novel coronavirus outbreak. Psychiatry Research, 286, 112895. https://doi.org/10.1016/j.psychres.2020.112895.

Publisher's Note Springer Nature remains neutral with regard to jurisdictional claims in published maps and institutional affiliations. 